\title{
Hepatic resection versus transarterial chemoembolization for patients with Barcelona Clinic Liver Cancer intermediate stage Child-Pugh A hepatocellular carcinoma
}

\author{
YIN-NONG ZHAO ${ }^{1}$, YONG-QUAN ZHANG ${ }^{2}$, JIA-ZHOU YE ${ }^{1}$, XING LIU $^{1}$, HONG-ZHI YANG $^{2}$, \\ FENG-YUN CONG ${ }^{2}$, BANG-DE XIANG ${ }^{1}$, FEI-XIANG WU ${ }^{1}$, LIANG MA ${ }^{1}$, LE-QUN LI ${ }^{1}$ and HAI-HONG YE ${ }^{2}$ \\ ${ }^{1}$ Department of Hepatobilliary Surgery, Affiliated Tumor Hospital of Guangxi Medical University, \\ Nanning, Guangxi 530021; ${ }^{2}$ Department of Hepatobilliary Surgery, \\ The Affiliated Minzu Hospital of Guangxi Medical University, Nanning, Guangxi 530001, P.R. China
}

Received July 30, 2015; Accepted September 1, 2016

DOI: $10.3892 / \mathrm{etm} .2016 .3810$

\begin{abstract}
The present study aimed to compare the overall and recurrence-free survival rates following hepatic resection (HR) and transcatheter arterial chemoembolization (TACE) in patients with Barcelona Clinic Liver Cancer (BCLC) classified intermediate-stage Child-Pugh A hepatocellular carcinoma (HCC). A total of 443 patients were examined, among whom 274 underwent HR, whereas 169 received TACE. The overall survival, recurrence-free survival between groups and subgroups, and risk factors with respect to mortality and recurrence, were analyzed. The 1-, 3- and 5-year overall and recurrence-free survival rates were 70,46 and $37 \%$ and 73,52 , and $37 \%$, respectively after $\mathrm{HR}$, compared with 38,15 , and $12 \%$ and 44,25 and $16 \%$, respectively after TACE. Overall and recurrence-free survival rates were significantly increased following HR compared with TACE. Subgroup analysis in the multi-nodule group showed that the 1-,3- and 5-year overall survival rates were 68,38 and $30 \%$ after HR, compared with 36,10 and $0 \%$ following TACE. In the solitary tumor group, 1-, 3- and 5-year overall survival rates were 71, 50 and $38 \%$ after HR, and 41, 22 and 15\% after TACE. The overall survival rate after HR was significantly increased compared with that after TACE in the solitary tumor and multi-nodule
\end{abstract}

Correspondence to: Professor Le-Qun Li, Department of Hepatobilliary Surgery, Affiliated Tumor Hospital of Guangxi Medical University, 71 Hedi Road, Nanning, Guangxi 530021, P.R. China

E-mail: lequnlic@yeah.net

Professor Hai-Hong Ye, Department of Hepatobilliary Surgery, The Affiliated Minzu Hospital of Guangxi Medical University, 232 Mingxiu Road, Nanning, Guangxi 530001, P.R. China

E-mail: haihongye@yeah.net

Key words: hepatocellular carcinoma, hepatic resection, transarterial chemoembolization, overall survival rate, recurrence-free survival groups. The risk factors for mortality include solitary tumor diameter $>10 \mathrm{~cm}$, multi-nodules, serum albumin level $\geq 35 \mathrm{~g} / \mathrm{l}$, prothrombin time $>13 \mathrm{sec}$, alphafetoprotein levels $>400 \mathrm{ng} / \mathrm{ml}$, and patients with hepatitis B virus. Solitary tumor diameter $>10 \mathrm{~cm}$, multi-nodules, and hepatitis $\mathrm{B}$ virus $(\mathrm{P}<0.001)$ were found to be associated with higher recurrence of HCC. Overall and recurrence-free survival rates were improved after HR compared with those after TACE in BCLC stage B, Child-Pugh A, HCC patients.

\section{Introduction}

Hepatocellular carcinoma (HCC) is the fifth most frequently occurring malignant tumor, with 500,000 patients succumbing to the disease globally each year $(1,2)$. Although safe and efficient therapies, including radiofrequency ablation (RFA), transarterial chemoembolization (TACE), and percutaneous ethanol injection have been reported, surgical resection remains the predominant modality applied for the treatment of the majority of patients with HCC patients as a result of advantages, such as low mortality and recurrence rates (3).

Hepatic resection (HR) is based on the tumor stage and the possibility of the preservation of liver function. In accordance with clinicopathologic parameters, several prognostic systems for HCC have been proposed. The most widely known and assessed systems adopted in various countries and areas are TNM (4), Okuda stage (5), Cancer of the Liver Italian Program (CLIP) (6,7), Chinese University Prognostic Index (8) and Barcelona Clinic Liver Cancer (BCLC) (9) and estrogen receptor classification (10). With an advanced capability to consider factors with respect to tumor stage, liver function and physical status, and its provision of treatment recommendations for the corresponding stage, the BCLC classification system is the most widely accepted treatment algorithm (11). Typically, HR is only favorable for the treatment of patients with early-stage $\mathrm{HCC}$ who have good liver function, as identified by a single nodule $\leq 5 \mathrm{~cm}$ or up to 3 nodules $\leq 3 \mathrm{~cm}$, Child-Pugh A or B and a performance score of 0 (10). RFA and liver transplantation are recommended for patients at the early stage but with poor liver function [portal hypertension/increased 
total bilirubin (T.BIL)] (12). However, for intermediate-stage patients (a single nodule $>5 \mathrm{~cm}$ or multi-nodular tumors; Child-Pugh A or B a with a performance score of 0 , no portal hypertension and distant metastasis) (10), TACE is indicated as the first-choice treatment. With the exception of the Barcelona group, several groups have recommended that BCLC stage B HCC should be considered a contraindication for $\operatorname{HR}(11,13)$. However, a number of authors argue that HR offers advantages with respect to patients with large tumors $(>5 \mathrm{~cm}$, and as large as $>10 \mathrm{~cm})$ and those with multi-nodular HCC $(14,15)$. As a result of the variation in recommendations, the role of surgical resection remains controversial.

The present retrospective study was conducted to clarify the role of surgical resection and evaluate the long-term and recurrence-free survival rates of HR compared with TACE in patients with BCLC intermediate-stage Child-Pugh A HCC.

\section{Patients and methods}

Patients. The database from the Affiliated Tumor Hospital and the Affiliated Minzu Hospital of Guangxi Medical University (Nanning, China) was retrospectively reviewed. A total of 1,340 patients with HCC were examined between January 2003 and December 2008. Among the patients analyzed, 669 were diagnosed to be under the resectable intermediate stage (BCLC stage B) and underwent HR or TACE as an initial treatment according to their preference, once they were informed of the surgical risks and presented with the guidelines for HCC treatment. The present study was conducted in accordance with the Declaration of Helsinki, and approval was obtained from the Ethics Committee of the Affiliated Minzu Hospital of Guangxi Medical University. Written informed consent was obtained from all participants.

Among the 669 patients, 323 patients who had Child-Pugh B liver function $(n=70)$, had undergone treatment with RFA $(n=26)$ and pre-operative TACE $(n=45)$ or who were not followed up $(n=82)$, were excluded. Subsequently, 443 patients with resectable intermediate-stage $\mathrm{HCC}$ with Child-Pugh A liver function were included, among whom 235 patients with solitary tumor and 9 patients with multi-nodules underwent treatment with HR, and 144 patients with solitary tumor and 25 patients with multi-nodules received TACE.

Diagnosis for all patients with HCC was based on the post-operative pathological findings or the diagnosis and treatment of primary liver cancer standardization expert consensus guidelines as follows: i) Concordant results obtained from two radiology techniques with contrast enhancement in the arterial phase and washout in the venous phase; and ii) typical image findings using an imaging technique, with an alphafetoprotein (AFP) level of $>400 \mathrm{ng} / \mathrm{ml}$.

Surgical resection. Indications for surgery in patients with BCLC stage B Child-Pugh A HCC were based on tumor location, number of tumors, absence of ascites, T.BIL $<3 \mathrm{mg} / \mathrm{dl}$ modified through Makuuchi's principle (16), and an appropriate amount of residual liver volume evaluated by volumetric CT, as previously described (17). Surgery was performed under general anesthesia through a bilateral subcostal incision with midline extension or with reversed L-shaped laparotomy. Intra-operative ultrasound was routinely performed. HR with a resection margin of $\leq 1 \mathrm{~cm}$ was performed, through which the primary tumor was eradicated and no residual tumor remained. Portal vein tumor thrombus, bile duct thrombus, hepatic vein tumor thrombus, and inferior vena cava tumor thrombus were not detected. The Pringle maneuver was used for 15 to $20 \mathrm{~min}$ and a 5 min clamp-free interval was performed to reduce blood loss. One or two 10 Fr Jackson-Pratt wound drains were applied after surgery.

TACE. Indications for TACE in patients with BCLC stage B Child-Pugh A HCC were based on the tumor location, number of tumors, absence of ascites, and T.BIL $<3 \mathrm{mg} / \mathrm{dl}$. TACE was performed subsequent to a diagnostic hepatic angiography. Contrast medium was injected into the arteries via a 4.1-French $\mathrm{RC} 1$ catheter, which was introduced into the abdominal aorta via the right superficial femoral artery using the Seldinger technique. The number, location, and size of tumors, as well as the arterial branches supplying the tumors, were identified. Iodized oil (10-20 ml), gelfoam particles with doxorubicin (30-50 mg) and cis-platinum (50-100 mg) were injected into the arterial branches. A follow-up computed tomography (CT) scan after 3 weeks in order to evaluate the effect of TACE. The TACE procedure was repeated if the tumors did not shrink and if liver function was satisfactory. The number of TACE cycles varied between 1 and 7 cycles.

Follow-up. Serum AFP was routinely measured, and B-mode ultrasound, dynamic CT, or MRI was performed a month after surgery or TACE. The possibility of recurrence was regularly monitored by measuring serum AFP levels every 1 and 3 months. Suspected intrahepatic recurrence was confirmed by dynamic CT and MRI or angiography. Equivocal situations were confirmed by pathological diagnosis through imaging-guided fine-needle aspiration cytology. Patients with recurrence were treated by TACE, RFA, or HR, regardless of their initial treatments. All patients were followed-up until April 2012.

Statistical analysis. Data were statistically analyzed using SPSS statistical software (version 18.0; SPSS, Inc., Chicago, IL, USA). Baseline characteristics between the two groups were compared using the Student's t-test and chi-square test. Survival time was defined as the period between the date of the initial HR or TACE and the date the patient succumbed to HCC, or as the end of the study for patients who survived. Recurrence-free survival was defined as the time from the date of the initial HR or TACE until the date of recurrence, or as the end of the study for patients who did not experience recurrence. Survival rates were estimated using the Kaplan-Meier method and compared using the log-rank test. The relative risks of mortality and recurrence were calculated using Cox's regression model and were compared using the log-rank test. $\mathrm{P}<0.05$ was considered to indicate a statistically significant difference.

\section{Results}

Baseline characteristics. Baseline characteristics of the 443 patients who underwent HR $(n=274)$ and TACE $(n=169)$ were determined and are presented in Table I. No difference in 
Table I. Characteristics and preoperative demographic data for patients with BCLC-B stage Child A HCC patients in the HR and TACE groups.

\begin{tabular}{lccr}
\hline & HR $(\mathrm{n}=274)$ & TACE $(\mathrm{n}=169)$ & P-value \\
\hline Age (years) & $45.7 \pm 11.9$ & $49.2 \pm 12.7$ & 0.144 \\
Male/female & $260(94.9 \%) / 14(5.1 \%)$ & $161(95.4 \%) / 8(4.6 \%)$ & 0.905 \\
Tumor size $(\mathrm{cm})$ & $8.2 \pm 2.4$ & $8.9 \pm 2.7$ & 0.101 \\
Tumor number & & & 0.234 \\
$=1$ & $235(85.8 \%)$ & $144(85.2 \%)$ & \\
$>1$ & $39(14.2 \%)$ & $25(14.8 \%)$ & 0.872 \\
Total bilirubin (umol/l) & $13.5 \pm 5.6$ & $14.6 \pm 7.3$ & 0.585 \\
Serum albumi $(\mathrm{g} / \mathrm{l})$ & $39.4 \pm 5.8$ & $38.7 \pm 5.4$ & 0.250 \\
Alanineamino transferase $(\mathrm{U} / \mathrm{l})$ & $49.1 \pm 25.4$ & $57.2 \pm 25.1$ & 0.527 \\
Platelet count $\left(\mathrm{x} 10^{9}, \mathrm{~g} / \mathrm{l}\right)$ & $208.6 \pm 80.7$ & $196.9 \pm 88.8$ & 0.879 \\
Prothrombin time(s) & $13.3 \pm 1.7$ & $13.1 \pm 1.8$ & 0.736 \\
Alphafetoprotein $(\mathrm{ng} / \mathrm{ml})$ & & & \\
$\geq 400$ & $155(56.8 \%)$ & $72(57.7 \%)$ & 0.778 \\
$<400$ & $119(43.2 \%)$ & & \\
Hepatitis B virus & & $137(81.3 \%)$ & $62(19.7 \%)$ \\
$(+)$ & $228(83.2 \%)$ & & \\
$(-)$ & $56(16.8 \%)$ & & \\
\hline
\end{tabular}

BCLC, Barcelona Clinic Liver Cancer; HCC, hepatocellular carcinoma; HR, hepatic resection; TACE, transcatheter arterial chemoembolization.

age, gender, tumor size, tumor number, T.BIL, albumin (ALB), alanine transaminase, blood platelet, prothrombin time, AFP, and hepatitis B surface antigen was found between the HR and TACE groups.

Survival. The 1-, 3- and 5-year overall survival rates and recurrence-free survival rates were 70,46 and $37 \%$, and 73, 52 and $37 \%$ in the HR group compared with 38,15 and $12 \%$, and 44, 25 and $16 \%$, respectively, in the TACE group. The respective mean overall and recurrence-free survival periods were 31.9 and 32.6 months in the HR group, and 14.3 and 13.4 months in the TACE group. Overall survival ( $\mathrm{P}<0.0001$; Fig. 1) and recurrence-free survival rates $(\mathrm{P}<0.0001$; Fig. 2$)$ were significantly increased in the HR group, compared with the TACE group.

Overall survival was also analyzed in the subgroups. In the multi-nodule group, the respective 1-, 3-, and 5-year overall survival rates were 68,38 and $30 \%$ in the HR group, compared with 36, 10 and $0 \%$ in the TACE group. Mean overall survival periods for the HR and TACE groups were 28.1 and 11.5 months, respectively. In the solitary tumor group, the respective 1-, 3-, and 5-year overall survival rates were 71, 50 and $38 \%$ in the HR group compared with 41,22 and $15 \%$ in the TACE group. Mean overall survival periods for the HR and TACE groups were 32.6 and 14.8 months, respectively. Overall survival in both the solitary (Fig. 3) and multi-nodule tumor groups (Fig. 4) was significantly longer after HR compared with that of patients who underwent TACE (both $\mathrm{P}<0.0001$ ).

Prognostic factors. Cox regression analysis indicated that the following were independent risk factors for survival: Solitary tumor diameter $>10 \mathrm{~cm}$, the presence of multi-nodules, serum ALB levels $\geq 35 \mathrm{~g} / 1$, prothrombin time $>13 \mathrm{sec}$, AFP levels $>400 \mathrm{ng} / \mathrm{ml}$, and hepatitis B virus. Therefore, solitary tumor diameter $>10 \mathrm{~cm}$, multi-nodules, hepatitis B virus, and TACE $(\mathrm{P}<0.001)$ are associated with higher recurrence of HCC (Tables II and III).

\section{Discussion}

The findings of the present study showed that the respective 1-, 3 - and 5-year overall survival rates were 70,46 and 37\% in the HR group, compared with 38,15 and $12 \%$ in the TACE group. The mean overall survival period was 31.9 months in the HR group, compared with 14.3 months in the TACE group. In the subgroup analysis, the 1-, 3- and 5-year overall survival rates were longer after HR than after TACE in both the solitary tumor group (HR, 71, 50, and 38\%; TACE, 41, 22, and 15\%) and the multi-nodule group (HR, 68, 38, and 30\%; TACE, 36, 10 and $0 \%$.

A higher recurrence rate of $\mathrm{HCC}$ was observed in the TACE group compared with the HR group. The respective 1-, 3- and 5 -year recurrence-free survival rates were 73,52 and $37 \%$ in the HR group, compared with 44, 25 and $16 \%$ in the TACE group. The mean recurrence-free survival period was 32.6 months in the HR group compared with 13.4 months in the TACE group. Both overall survival and recurrence-free survival rates were significantly longer in the HR group compared with those of the TACE group. Therefore, these findings indicated that HR may be superior to TACE for the treatment of patients with BCLC intermediate stage Child-Pugh A HCC. However, no difference was observed in the mortality rates during 


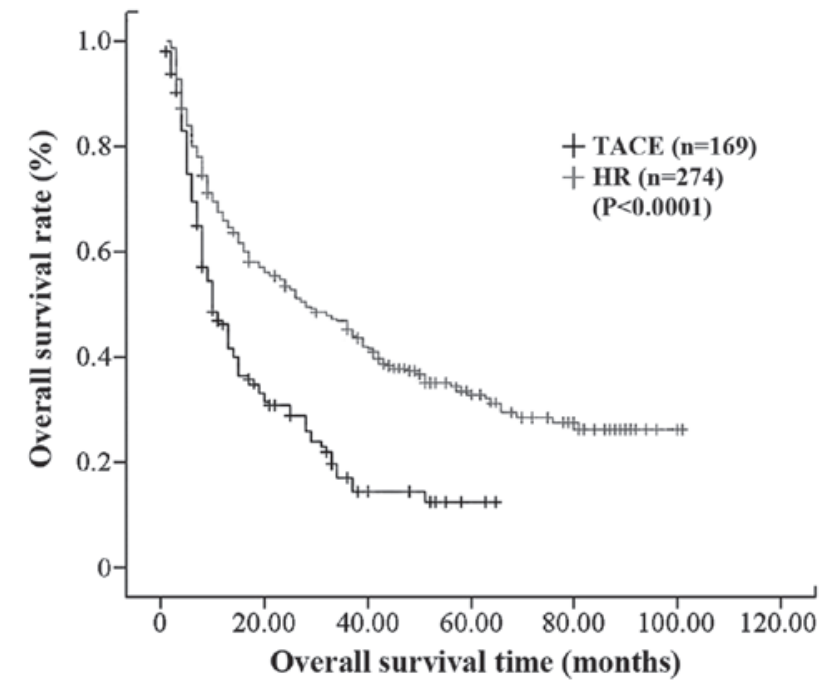

Figure 1. Overall survival curves in the TACE and HR groups. TACE, transcatheter arterial chemoembolization; HR, hepatic resection.

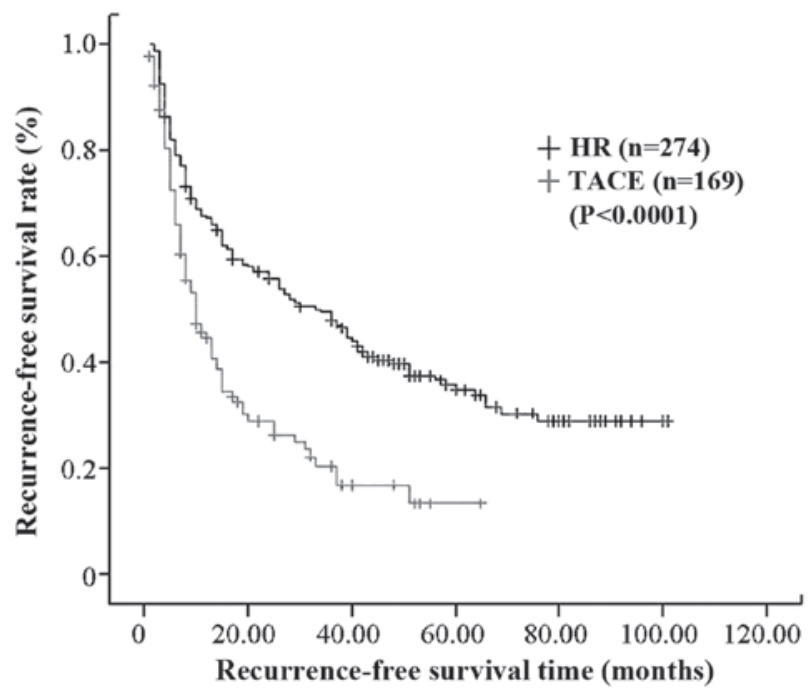

Figure 2. Recurrence-free survival curves in the TACE and hepatic resection groups. TACE, transcatheter arterial chemoembolization; HR, hepatic resection.

hospitalization between the two groups. The present study found that the treatment modality was an independent prognostic factor of overall survival based on the Cox regression model (HR vs. TACE, RR=1.001; $\mathrm{P}=0.025$ ).

The liver's blood supply is provided by the portal vein and hepatic artery. Typically, $\sim 75 \%$ of the blood in the liver is supplied from portal vein, whereas the remaining $25 \%$ arrives through the hepatic artery. However, $95-99 \%$ of blood in HCC is supplied by the hepatic artery, and only a small amount is provided by the portal vein $(18,19)$. Iodized oil $(10-20 \mathrm{ml})$, gelfoam particles with doxorubicin $(30-50 \mathrm{mg})$, and cis-platinum (50-100 mg) were injected into the arterial branches via TACE in the present study. Based on the fact that lipiodol selectively accumulates in tumors when delivered intra-arterially and is a carrier for anti-cancer drugs, TACE is able to provide direct therapeutic efficacy by blocking vessels that deliver nutrients to tumors, and providing large

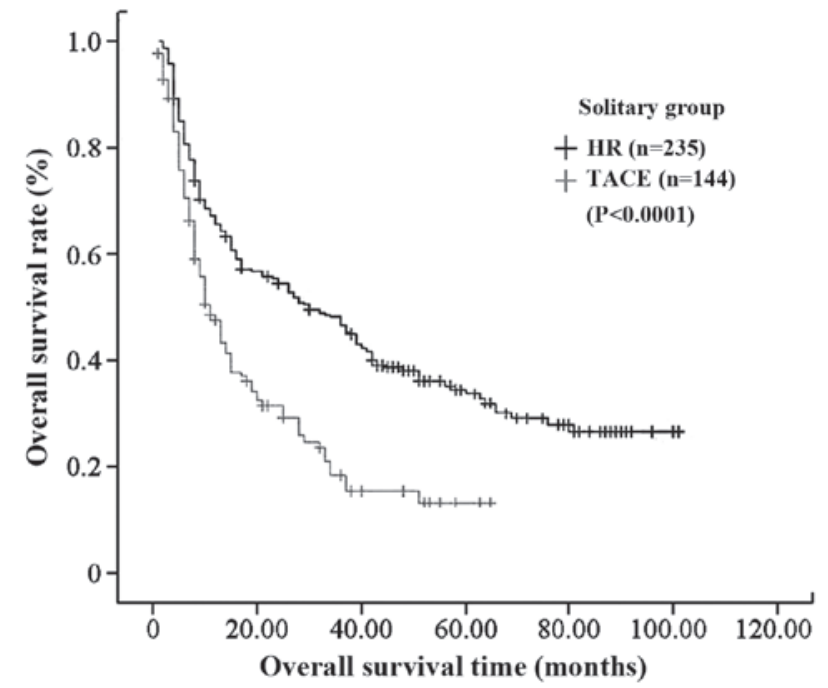

Figure 3. Overall survival curves following TACE or HR in the solitary tumor group. TACE, transcatheter arterial chemoembolization; HR, hepatic resection.

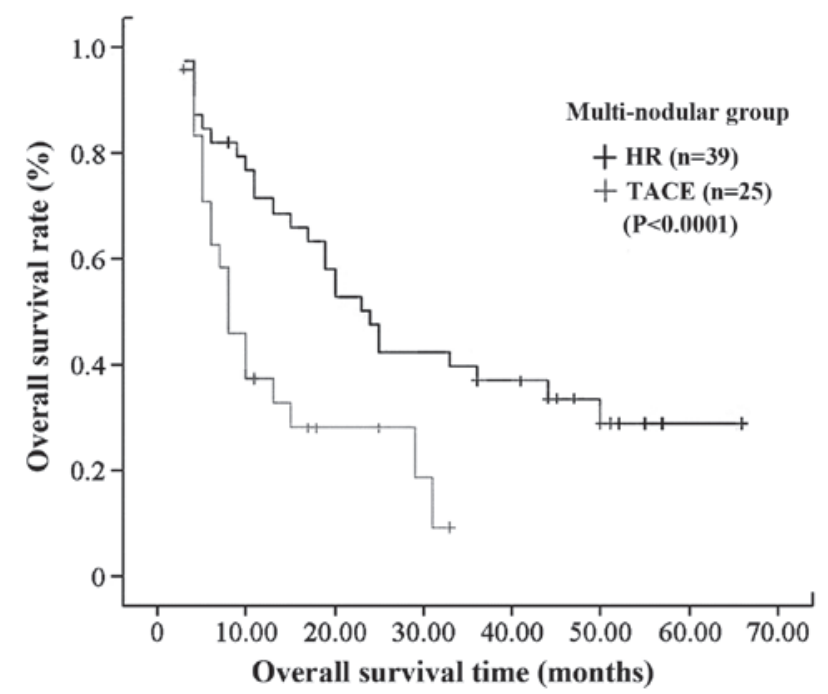

Figure 4. Overall survival curves following TACE or HR in the multi-nodular group. TACE, transcatheter arterial chemoembolization; HR, hepatic resection.

doses of sustainable chemotherapy without affecting normal liver cells (20-22). However, TACE is only a palliative treatment, as the vessels delivering nutrients to tumors are not able to be completely blocked as a result of the nutrient vessels from the portal vein. Thus, tumor necrosis is not completely achieved. The risk of tumor recurrence increases as a result of tumor blood vessel collateral circulation. Llovet et al (23) revealed that TACE induces extensive tumor necrosis in $30-50 \%$ of patients; however, a complete response has only been achieved in $<2 \%$ of patients. Whether TACE is able to provide a long-term survival benefit has yet to be elucidated, however a number of authors have reported the 5-year overall survival rates for patients with HCC who underwent TACE to be between 6 and 19\% $(24,25)$, which is similar to the findings of the present study (12\%). However, it has been suggested that repetitive TACE may damage the remnant liver parenchyma, 
Table II. Cox regression model for factors associated with survival and recurrence for patients included in the study.

\begin{tabular}{|c|c|c|c|}
\hline Pre-operative demographic data & RR value & $95 \%$ for CI & P-value \\
\hline \multicolumn{4}{|l|}{ Mortality } \\
\hline Age ( $\geq 60$ vs. $<60$ years $)$ & 0.998 & $0.989-1.008$ & 0.754 \\
\hline Male vs. female & 0.932 & $0.902-0.981$ & 0.644 \\
\hline Tumor size (>10 vs. $\leq 10 \mathrm{~cm})$ & 1.087 & $1.039-1.137$ & $<0.0001$ \\
\hline Tumor number (=1 vs. $>1)$ & 1.045 & $1.012-1.086$ & 0.025 \\
\hline Total bilirubin $(>17.1 \mathrm{vs.} \leq 17.1 \mathrm{umol} / \mathrm{l})$ & 1.013 & $0.993-1.034$ & 0.191 \\
\hline Serum albumin $(\geq 35$ vs. $<35 \mathrm{~g} / \mathrm{l})$ & 0.975 & $0.952-0.997$ & 0.028 \\
\hline Alanine amino transferase ( $>40 \mathrm{vs} . \leq 40 \mathrm{U} / 1$ ) & 1.000 & $0.997-1.002$ & 0.752 \\
\hline Platelet count ( $>100$ vs. $\left.\leq 100 \times 10^{9} \mathrm{~g} / \mathrm{l}\right)$ & 1.000 & $0.998-1.001$ & 0.756 \\
\hline Prothrombin time (>13 vs. $\leq 13 \mathrm{sec})$ & 1.033 & $1.001-1.067$ & 0.045 \\
\hline Alphafetoprotein (>400 vs. $<400$ ng/ml) & 0.965 & $0.921-1.001$ & 0.041 \\
\hline Hepatitis B virus (+ vs. -) & 0.982 & $0.936-1.082$ & 0.031 \\
\hline Hepatic resection vs. TACE & 1.001 & $0.998-1.003$ & 0.025 \\
\hline \multicolumn{4}{|l|}{ Recurrence } \\
\hline Age $(\geq 60$ vs. $<60$ years $)$ & 1.002 & $0.971-1.008$ & 0.631 \\
\hline Male vs. female & 0.941 & $0.903-0.981$ & 0.069 \\
\hline Tumor size, $\mathrm{cm}(>10$ vs. $\leq 10 \mathrm{~cm})$ & 1.063 & $0.989-1.116$ & $<0.0001$ \\
\hline Number of tumors (=1 vs. $>1$ ) & 1.042 & $0.982-1.098$ & $<0.0001$ \\
\hline Total bilirubin (>17.1 vs. $\leq 17.1 \mathrm{umol} / \mathrm{l})$ & 0.951 & $0.932-0.993$ & 0.326 \\
\hline Serum albumin $(\geq 35$ vs. $<35 \mathrm{~g} / \mathrm{l})$ & 1.003 & $0.986-1.009$ & 0.052 \\
\hline Alanine amino transferase (>40 vs. $\leq 40 \mathrm{U} / \mathrm{l})$ & 0.984 & $0.961-1.020$ & 0.491 \\
\hline Platelet count (>100 vs. $\left.\leq 100 \times 10^{9} \mathrm{~g} / \mathrm{l}\right)$ & 1.005 & $0.997-1.012$ & 0.738 \\
\hline Prothrombin time (>13 vs. $\leq 13 \mathrm{sec}$ ) & 0.966 & $0.939-0.994$ & 0.352 \\
\hline Alphafetoprotein (>400 vs. $<400$ ng/ml) & 0.977 & $0.935-1.015$ & 0.361 \\
\hline Hepatitis B virus (+ vs. -) & 0.962 & $0.922-0.981$ & 0.048 \\
\hline Hepatic resection vs. TACE & 1.065 & $0.971-1.111$ & $<0.001$ \\
\hline
\end{tabular}

RR, risk ratio; CI, confidence interval; TACE, transcatheter arterial chemoembolization.

particularly in cirrhotic patients, which may result in the impairment or deterioration of liver function $(26,27)$.

By contrast, with the advances made in terms of surgical techniques and pre-operative care, HR remains widely accepted as the standard modality for the treatment of the majority of HCC patients, as it improves their long-term survival (28). A number of scholars believe that patients with HCC with sufficient preservation of the liver function may still be cured $(13,29-31)$. Assessment of the residual liver function is a crucial indicator for HR. HR has been performed in patients with BCLC intermediate stage large and multi-nodular HCC. Lin et al (29) reported the 3-year survival rate of patients with BCLC stage B HCC after initial HR to be $49 \%$ compared with $2 \%$ for patients who underwent TACE. However, the patients in the TACE group exhibited lower serum ALB and larger tumor sizes, resulting in a less favorable prognosis (29). $\mathrm{Ng}$ et al (11) revealed that HR is efficient and safe for the treatment of patients with $\mathrm{HCC}$ with a diameter of $>5 \mathrm{~cm}$ or multiple -nodules $(>3)$. Furthermore, the 5-year survival rate for HCC patients who underwent HR was $39 \%$ (13), which was similar to the findings of the present study. Ho et al (30) suggested that HR was able to prolong the survival of patients with BCLC-B HCC with multi-nodules compared with TACE.
Wang et al (31) retrospectively analyzed the survival time of patients with intermediate stage $\mathrm{HCC}$, and found the 5-year survival rates for patients with HCC BCLC stage-B after initial HR to be $50.5 \%$, compared with $16.9 \%$ in patients who received TACE.

The BCLC classification encompasses the tumor stage, liver function, and physical status, as well as the recommendation of treatment modalities for the corresponding stages and is accepted as one of the most reliable staging and treatment strategy systems for HCC (9). Considering the high risk of hepatic decompensation after surgical resection, patients with BCLC intermediate-stage HCC are not suitable candidates for HR. In particular, the livers of patients with cirrhosis have a reduced effective remnant liver volume of regenerative reserve. In the present study, CT or MRI was employed to measure the remnant liver volume of patients with BCLC intermediate stage Child-Pugh A HCC who may be classified as having resectable HCC (20). HR was performed in patients with multi-modal and large tumors $(>5 \mathrm{~cm})$, or if the tumor size was $>10 \mathrm{~cm}$ after volumetric CT and MRI scans. With the advancements made with respect to pre-operative measurements and surgical techniques, the current results indicate that $\mathrm{HR}$ is a promising option in patients with BCLC intermediate 
Table III. Cox regression model for factors associated with the survival and recurrence of hepatic cellular carcinoma in patients included in the present study.

\begin{tabular}{lccc}
\hline Pre-operative demographic data & RR value & 95\% CI & P-value \\
\hline Mortality & & & $<0.0001$ \\
Tumor size ( $>10$ vs. $\leq 10 \mathrm{~cm})$ & 1.087 & $1.039-1.137$ & 0.025 \\
Number of tumors $(=1$ vs. $>1)$ & 1.045 & $1.012-1.086$ & 0.028 \\
Serum albumin $(\geq 35$ vs. $<35 \mathrm{~g} / \mathrm{l})$ & 0.975 & $0.952-0.997$ & 0.045 \\
Prothrombin time $(>13$ vs. $\leq 13 \mathrm{sec})$ & 1.033 & $1.001-1.067$ & 0.041 \\
Alphafetoprotein $(>400$ vs. $<400 \mathrm{ng} / \mathrm{ml})$ & 0.965 & $0.921-1.001$ & 0.031 \\
Hepatitis B virus (+ vs. -$)$ & 0.982 & $0.936-1.082$ & 0.025 \\
Hepatic resection vs. TACE & 1.001 & $0.998-1.003$ & $<0.0001$ \\
Recurrence & & & $<0.0001$ \\
Tumor size $(>10$ vs. $\leq 10 \mathrm{~cm})$ & 1.063 & $0.989-1.116$ & 0.048 \\
Tumor number $(=1$ vs. $>1)$ & 1.042 & $0.982-1.098$ & $<0.001$ \\
Hepatitis B virus $(+$ vs. -$)$ & 0.962 & $0.922-0.981$ & $0.971-1.111$ \\
Hepatic resection vs. TACE & 1.065 & & \\
\hline
\end{tabular}

$\mathrm{RR}$, risk ratio; CI, confidence interval; TACE, transcatheter arterial chemoembolization.

stage Child-Pugh A HCC who have adequate liver function preservation. In particular, HR improves the survival-time and has a lower recurrence rate compared with TACE.

In the present study, Cox regression model analysis revealed that a solitary tumor diameter $>10 \mathrm{~cm}$, multi-nodules, serum ALB level $\geq 35 \mathrm{~g} / \mathrm{l}$, prothrombin time $>13 \mathrm{sec}$, AFP level $>400 \mathrm{ng} / \mathrm{ml}$, infection with hepatitis B virus, cirrhosis, and the treatment modality (TACE) were independent risk factors for poor survival. Solitary tumor diameter $>10 \mathrm{~cm}$, multi-nodules, hepatitis B virus, and TACE were found to be associated with high recurrence. The use of TACE as a therapeutic intervention and predictor of recurrence has generated widespread interest. Xia et al (32) indicated that the serum levels of angiogenic factors in post-TACE patients were significantly higher than those of post-operative patients. Serum levels of vascular endothelial cells in patients post-TACE were found to be significantly lower compared with those of post-operative patients. By contrast, HR was more efficacious in terms of preventing the renascence of vessels and recurrence of HCC.

Although the present study was conducted across two centers, limitations such as selection bias in terms of the patients' choice of treatment between HR and TACE, the different stages of liver function and the tumor location must be noted. Although the present study compared the baseline characteristics and pre-operative demographic data and found no significant difference between the groups with patients with the same stage of liver function, there may still be heterogeneity between the patients (33). Multi-institutional prospective randomized control trials are required to clarify the role of HR for patients with BCLC intermediate stage Child-Pugh A HCC.

The present study demonstrated that HR was superior in terms of resulting in longer overall survival and recurrence-free survival compared with TACE in patients with BCLC intermediate stage Child-Pugh A HCC. Therefore, HR has the potential to be an alternative treatment applied to selected patients with BCLC intermediate stage Child-Pugh A HCC.

\section{References}

1. Bosh FX, Ribes $\mathrm{J}$ and Borràs $\mathrm{J}$ : Epidemiology of primary liver cancer. Semin Liver Dis 19: 271-285, 1999.

2. Kamangar F, Dores GM and Anderson WF: Patterns of cancer incidence, mortality, and prevalence across five continents: Defining priorities to reduce cancer disparities in different geographic regions of the world. J Clin Oncol 24: 2137-2150, 2006.

3. Taketomi A, Kitagawa D, Itoh S, Harimoto N, Yamashita Y, Gion T, Shirabe K, Shimada M and Maehara Y: Trends in morbidity and mortality after hepatic resection for hepatocellular carcinoma: An institute's experience with 625 patients. J Am Coll Surg 204: 580-587, 2007.

4. Vauthey JN, Lauwers GY, Esnaola NF, Do KA, Belghiti J, Mirza N, Curley SA, Ellis LM, Regimbeau JM, Rashid A, et al: Simplified staging for hepatocellular carcinoma. J Clin Oncol 20: 1527-1536, 2002.

5. Okuda K, Ohtsuki T, Obata H, Tomimatsu M, Okazaki N, Hasegawa H, Nakajima Y and Ohnishi K: Natural history of hepatocellular carcinoma and prognosis in relation to treatment. Study of 850 patients. Cancer 56: 918-928, 1985.

6. Kudo M, Chung H and Osaki Y: Prognostic staging system for hepatocellular carcinoma (CLIP score): Its value and limitations, and a proposal for a new staging system, the Japan Integrated Staging Score (JIS score). J Gastroenterol 38: 207-215, 2003.

7. A new prognostic system for hepatocellular carcinoma: A retrospective study of 435 patients: The Cancer of the Liver Italian Program (CLIP) investigators. Hepatology 28: 751-755, 1998.

8. Leung TW, Tang AM,Zee B, Lau WY, Lai PB, Leung KL, Lau JT, Yu SC and Johnson PJ: Construction of the Chinese University Prognostic Index for hepatocellular carcinoma and comparison with the TNM staging system, the Okuda staging system and the Cancer of the Liver Italian Program staging system: A study based on 926 patients. Cancer 94: 1760-1769, 2002.

9. Llovet JM, Brú C and Bruix J: Prognosis of hepatocellular carcinoma: The BCLC staging classification. Semin Liver Dis 19: 329-338, 1999.

10. Villa E, Colantoni A, Cammà C, Grottola A, Buttafoco $\mathrm{P}$, Gelmini R, Ferretti I and Manenti F: Estrogen receptor classification for hepatocellular carcinoma: Comparison with clinical staging systems. J Clin Oncol 21: 441-446, 2003.

11. El-Serag HB, Marrero JA, Rudoplph L and Reddy KR: Diagnosis and treatment of hepatocellular carcinoma. Gastroenterology 134: $1752-1763,2008$

12. Bruix T and Llovet JM: Prognostic prediction and treatment strategy in hepatocellular carcinoma. Hepatology 35: 519-524, 2002. 
13. Ng KK, Vauthey JN, Pawlik TM, Lauwers GY, Regimbeau JM, Belghiti J, Ikai I, Yamaoka Y, Curley SA, Nagorney DM, et al: Is hepatic resection for large and multinodular hepatocellular carcinoma justified? Results from a multi-institutional database. Ann Surg Oncol 12: 364-373, 2005.

14. Hanazaki K, Kajikawa S, Shimozawa N, Shimada K, Hiraguri M, Koide N, Adachi W and Amano J: Hepatic resection for hepatocellular carcinomain diameter of $>0 \mathrm{or}=10 \mathrm{~cm}$. Hepatogastroenterology 49: 518-523, 2002.

15. Poon RT, Fan ST and Wong J: Selection criteria for hepatic resection in patients with large hepatocellular carcinoma larger than $10 \mathrm{~cm}$ in diameter. J Am Coll Surg 194: 592-602, 2002.

16. Machuuli M, Hasegawa $\mathrm{H}$ and Yamazaki S: Ulitrasonically guided subsegmentectomy. Surg Gynecol Obstet 161: 346-350, 1985

17. Hsieh CB, Yu CY, Tzao C, Chu HC, Chen TW, Hsieh HF, Liu YC and Yu JC: Prediction of the risk of hepatic failure in patients woth portal vein invasion hepatoma after hepatic resection. Eur J Surg Oncol 32: 72-76, 2006

18. Roayaie S, Frischer JS, Emre SH, Fishbein TM, Sheiner PA Sung M, Miller CM and Schwartz ME: Long-term results with multimodal adjuvant therapy and liver transplantation for the treatment of hepatocellular carcinomas larger than 5 centimeters. Ann Surg 235: 533-539, 2002

19. Ueno K, Miyazono N, Inoue H, Nishida H, Kanetsuki I and Nakajo M: Transcatheter arterial chemoembolization therapy using iodized oil for patients with unresectable hepatocellular carcinoma: Evaluation of three kinds of regimens and analysis of prognostic factors. Cancer 88: 1574-1581, 2000.

20. Lee DS and Seong J: Radiotherapeutic options for hepatocellular carcinoma with portal vein tumor thrombosis. Liver Cancer 3 : 18-30, 2014.

21. Katamura Y, Aikata H, Kimura Y, Kawaoka T, Takaki S, Waki K, Hiramatsu A, Kawakami Y, Takahashi S, Ishikawa M, et al: Intra-arterial 5-fluorouracil/interferon combination therapy for hepatocellular carcinoma with portal vein tumor thrombosis and extrahepatic metastases. J Gastroenterol Hepatol 25: 1117-1122, 2010.

22. Huang YH, Wu JC, Lui WY, Chau GY, Tsay SH, Chiang JH, King KL, Huo TI, Chang FY and Lee SD: Prospective case-controlled trial of adjuvant chemotherapy after resection of hepatocellular carcinoma. World J Surg 24: 551-555, 2000.

23. Llovet JM, Real MI, Montaña X, Planas R, Coll S, Aponte J, Ayuso C, Sala M, Muchart J, Solà R, et al: Arterial embolization or chemoembolization versus symptomatic treatment in patients with unresectable hepatocellular carcinoma: A randomized controlled trial. Lancet 359: 1734-1739, 2002.
24. O'Suilleabhain CB, Poon RT, Yong JL, Ooi GC, Tso WK and Fan ST: Factors predictive of 5-year survival after transarterial chemoembolization for inoperable hepatocellular carcinoma. $\mathrm{Br}$ J Surg 90: 325-331, 2003.

25. Ono Y, Yoshimasu T, Ashikaga R, Inoue M, Shindou H, Fuji K, Araki Y and Nishimura Y: Long-term results of lipiodol-transcatheter arterial embolization with cisplatin or doxorubicin for unresectable hepatocellular carcinoma. Am J Clin Oncol 23: $564-568,2000$

26. Ono T, Yamanoi A, Nazmy EL Assal O, Kohno $\mathrm{H}$ and Nagasue N: Adjuvant chemotherapy after resection for hepatocellular carcinoma causes deterioration of long-term prognosis in cirrhotic patients: Metaanalysis of three randomized controlled trials. Cancer 91: 2378-2385, 2001.

27. Chao AO, Yuen MF, Hui CK, Tso WK and Lai CL: A prospective study regarding the complication of transcatheter intraarterial lipiodol chemoembolization in patients with hepatocellular carcinoma. Cancer 94: 1747-1752, 2002.

28. Llovet JM and Bruix J: Systematic review of randomized trials for unresectable hepatocellular carcinoma: Chemoembolization improves survival. Hepatology 37: 429-442, 2003.

29. Lin CT, Hsu KF, Chen TW, Yu JC, Chan DC, Yu CY, Hsieh TY, Fan HL, Kuo SM, Chung KP and Hsieh CB: Comparing hepatic resection and transarterial chemoembolization for Barcelona Clinic Liver Cancer (BCLC) Stage B hepatocellular carcinoma: Change for treatment of choice? World J Surg 34: 2155-2161, 2010.

30. Ho MC, Huang GT, Tsang YM, Lee PH, Chen DS, Sheu JC and Chen $\mathrm{CH}$ : Liver resection improves the survival of patients with multiple hepatocellular carcinomas. Ann Surg Oncol 34: $848-855,2009$

31. Wang JH, Changchien CS, Hu TH, Lee CM, Kee KM, Lin CY, Chen CL, Chen TY, Huang YJ and Lu SN: The efficacy of treatment schedules according to Barcelona clinic liver cancer staging for hepatocellular carcinoma-Survival analysis of 3892 patients. Eur J Cancer 44: 1000-1006, 2008.

32. Xia HT, Guo GH, Huang XQ and Wang J: Variations in serum level of cytokines associated with vascular endothelial cells before and after treatment with either TACE and liver resection in patients with hepatocellular carcinoma. Chin J Hepatobiliary Surg 1: 23-26, 2012.

33. Lin CY, Kee KM, Wang JH, Lee CM, Chen CL, Changchien CS, $\mathrm{Hu} \mathrm{TH}$, Cheng YF, Hsu HC, Wang CC, et al: Is the cancer of the liver Italian program system an adequate weighting for survival of hepatocellular carcinoma? Evaluation of intrascore prognostic value among 36 subgroups. Liver Int 29: 74-81, 2009. 